\title{
Médecins et infirmières de la Suisse - surtout de Genève - dans les guerres des peuples yougoslaves
}

Par Zdenko Levental

Depuis plus d'un siècle, des centaines de médecins et d'infirmières suisses ont fait partie des corps de volontaires médicaux engagés dans de nombreuses guerres dans différents pays. Presque tous ont montré dans ce domaine d'activité des dispositions remarquables et un intérêt persistant. (Nous l'avons signalé déjà dans quelques de nos travaux : ${ }^{1,2,3}$.)

Comment interpréter ce phénomène ? Sans doute, plusieurs raisons et motivations ont joué un rôle. Sur le plan national, d'une part, l'engagement de missions médicales à l'étranger servait le besoin des responsables militaires d'obtenir des informations de première main sur de nouvelles armes et sur l'efficacité des méthodes récentes de traitement, sur l'organisation de soins et le transport des blessés ${ }^{4}$. Pour le Comité International de la Croix-Rouge et la Croix-Rouge Suisse d'autre part, il s'agissait de servir comme intermédiaires ou organisateurs directs d'actions d'aide et de veiller à l'application pratique de la Convention de Genève $^{5}$. Quant aux motifs individuels des volontaires, ils devaient être encore plus complexes: sans aucun doute, l'intérêt professionnel ou scientifique de faire ou de découvrir quelque chose de nouveau a joué un rôle considérable. Cependant, un élan de solidarité humaine, surtout vis-à-vis des petits peuples, un sentiment de sympathie politique ou d'identification religieuse ont certainement déterminé maintes décisions.

La tradition de ces engagements est longue : nous pouvons en suivre les traces à partir des champs de bataille d'Italie en 1859, à travers de nombreuses "petites» et grandes guerres du $\mathrm{XIX}^{\mathrm{e}}$ et $\mathrm{XX}^{\mathrm{e}}$ siècle jusqu'aux conflits armés de nos jours ${ }^{6}$.

Dans cette étude, nous sommes obligés de nous limiter aux actions entreprises par les Genevois et Genevoises dans les luttes de libération des peuples yougoslaves, bien que, lors de différentes guerres dans les Balkans, des missions suisses opéraient souvent simultanément dans les différents camps, pour prouver par là leur bonne foi et leur neutralité. Pendant le dernier tiers du XIX ${ }^{\mathrm{e}}$ siècle, la Grèce, la Bulgarie, la Serbie et le Monténégro, en train de se libérer de l'occupation turque qui a duré presque cinq siècles, ont réussi à mettre sur pied des forces étonnantes. Toutefois, leurs services sanitaires manquaient de cadres et leurs Croix-Rouges étaient encore trop faibles ou sans expériences pour être d'un soutien efficace pour l'armée. Cette insuffisance se faisait cruellement jour lorsque, après des batailles 
féroces, le nombre de blessés se montait à plusieurs milliers, et ceci en l'espace de quelques jours. Une aide étrangère devenait dès lors indispensable et urgente.

Les objectifs concrets, spécifiques, poursuivis par les actions de secours de l'étranger, variaient selon les besoins locaux et les problèmes qui prédominaient. La tâche qui importait en premier lieu était de renforcer les équipes des hôpitaux déjà existants ou d'organiser des lazarets de réserve, de s'occuper des blessés, des cas à opérer, des malades. Souvent il fallait aussi pourvoir aux besoins d'une population civile en détresse. Quelquefois, les maladies infectieuses s'en mêlaient ou devenaient même l'ennemi numéro un. Dans les deux guerres de 1912-1913 c'étaient les infections intestinales; en 1915, en Serbie, le typhus exanthématique. Par cette épidémie dévastatrice qui se caractérisa par une mortalité extrêmement haute, proche de $60 \%$, plusieurs centaines de milliers de civils, de soldats serbes et des prisonniers de guerre autrichiens furent touchés. Des centaines de médecins et d'infirmières succombèrent aux atteintes du typhus. Les missions étrangères, les Suisses inclus, ne furent pas épargnées.

Malgré la nécessité de nous concentrer ici sur le rôle des Genevois, il est difficile de se limiter strictement à cette ville et canton, car les membres des missions genevoises se recrutaient souvent aussi parmi les citoyens d'autres cantons romands. Quelquefois il s'agissait d'une vraie mobilisation des hommes de bonne volonté de la Suisse tout entière ${ }^{7}$, et il est presque impossible de ne pas mentionner ici certaines actions provenant de différents cantons.

L'année 1875 marque le départ de la première mission suisse et genevoise vers le territoire d'un des peuples yougoslaves. Fin décembre, le Comité International de la Croix-Rouge décida d'envoyer au Monténégro le jeune médecin Dr Frédéric Ferrière, le naturaliste Aloïs Humbert et le pharmacien Charles Goetz, tous les trois de Genève. Au cours de l'insurrection dans la province de l'Herzégovine contre la Turquie, plus que 60000 réfugiés civils et un grand nombre des guerriers blessés trouvèrent asile au Monténégro voisin, où, une fois de plus, se déclancha un conflit armé avec la Turquie. La mission du Comité International fut chargée de faciliter la création d'une Société de la Croix-Rouge du Monténégro, de veiller sur l'application de la Convention de Genève par les parties belligérantes et de participer aux soins aux blessés, dans ce pays minuscule, privé presque complètement de médecins. En plus d'un compte rendu sur leur séjour de trois mois et sur les tâches accomplies, publié 1876 par les délégués ${ }^{8}$, Ferrière a envoyé une série de lettres à un journal allemand, l'«Allgemeine Zeitung» d'Augsburg. Plus tard, il a écrit une étude détaillée sur le Monténégro, le pays, ses habitants, le folklore et sa médecine populaire, pour la revue genevoise de géographie Le Globe ${ }^{9}$. 


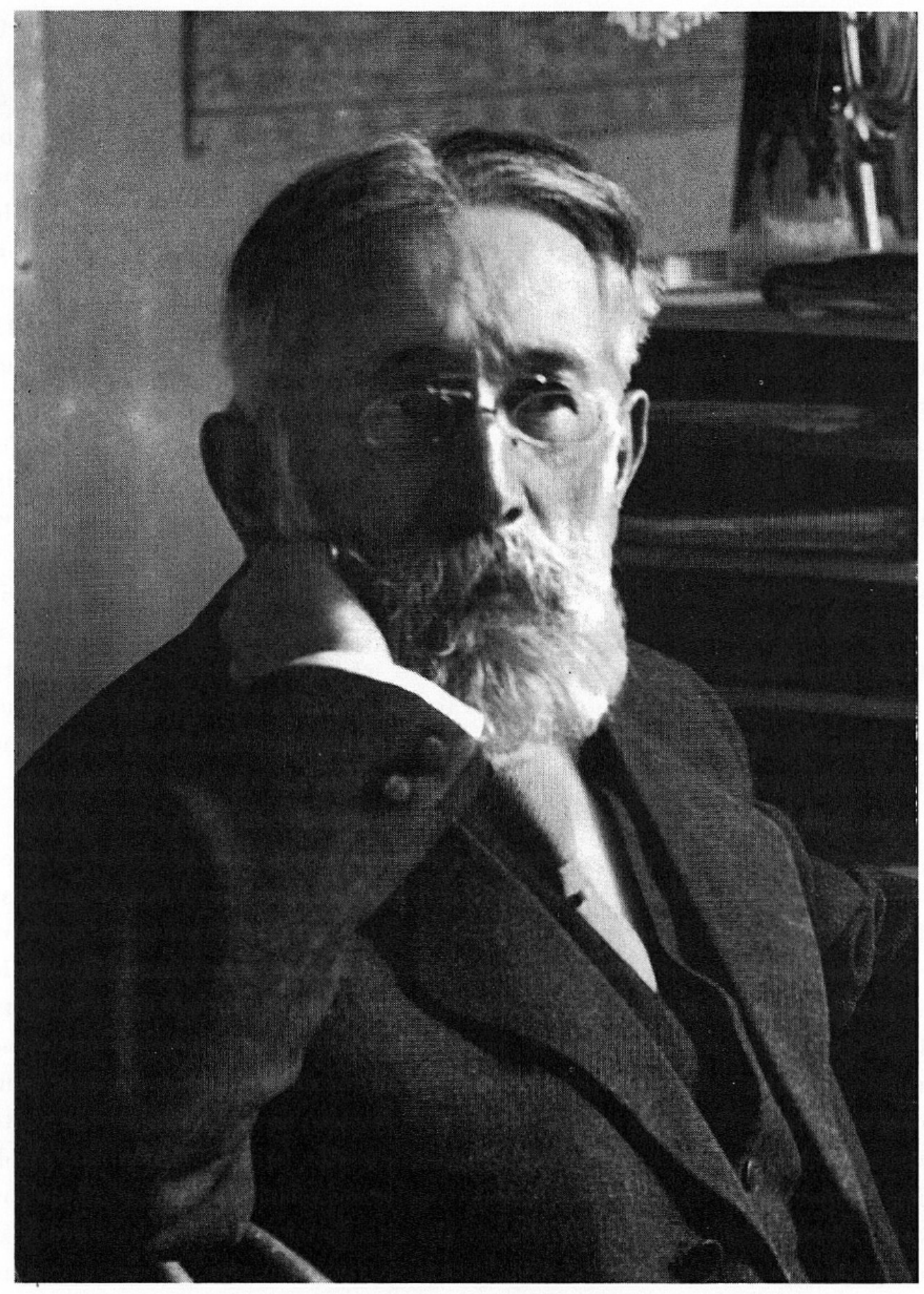

Fig. 1. Le Dr. Frédéric Ferrière - vers l'année 1915 
Ferrière - neveu d'Appia - qui en tant qu'étudiant en médecine, avait déjà participé activement aux transports de blessés lors de la guerre franco-allemande de 1870, devint par la suite un expert très connu, jusque sur le plan international, pour certains problèmes de la médecine militaire et de catastrophe, surtout pour les moyens de transport des blessés. Membre infatigable du Comité International de la Croix-Rouge, il a de nouveau visité les Balkans en 1913 et $1918^{10,11,12,13,14,15}$.

Le nom de Ferrière restera inscrit dans l'histoire mondiale de la charité grâce à un véritable chef-d'œuvre d'humanisme: en 1914-1918, en dépit de tous les obstacles et en l'absence d'une convention nécessaire, il a créé et développé à Genève une section spéciale de l'Agence internationale des prisonniers - qui s'occupait de la protection des populations civiles, sous occupation ennemie, souffrant de déportation ou d'émigration. Deux grands écrivains et pacifistes de cette époque, Romain Rolland ${ }^{16}$ et Stefan Zweig ${ }^{17}$, ont rendu hommage à cette œuvre de Ferrière.

En 1877, lorsque encore une fois les armes parlent entre la Turquie, la Serbie et le Monténégro, le Comité International a installé une Agence internationale à Trieste, ayant pour but de coordonner ses activités et celles de la CroixRouge de différents pays, aussi près que possible de la région des hostilités. De nouveau, c'est un médecin genevois, le célèbre professeur Adolphe d'Espine, qui fut chargé en tant que secrétaire du Comité International, de veiller à son bon fonctionnement.

Au cours des deux guerres des Balkans de 1912 et 1913, plus de 80 médecins et infirmières suisses se sont rendus dans les quatres pays belligérants ${ }^{18,19,20}$. La plus grande ambulance fut formée sur l'initiative du professeur César Roux de Lausanne et comprenait huit médecins et onze infirmières originaires des cantons de Vaud et de Genève. Ce groupe, à la tête duquel se trouvait le chirurgien genevois Albert Reverdin (frère de Jaques-Louis Reverdin), soigna en Epire, au nord de la Grèce, environ 2300 blessés ${ }^{21}$. Ce chiffre élevé - atteint aussi par d'autres missions - révèle quelles étaient les possibilités des médecins suisses d'accumuler d'importantes nouvelles expériences et de les comparer plus tard avec celles publiées dans les revues médicales d'autres pays. L'échange d'observations et d'interprétations dans un cadre international se déroule aussi par la voie personnelle et directe, comme ce fut le cas pour Alfred-C. Matthey ${ }^{22}$, qui a communiqué les résultats de son travail en Serbie et à Constantinople aux membres de la Société de médecins de Danzig.

De précieuses observations et propositions pour une meilleure organisation des soins aux blessés se trouvent dans le rapport de l'envoyé du Comité International, le major Carle de Marval, de Neuchâtel, haut fonctionnaire de la Croix-Rouge Suisse dans les pays balkaniques ${ }^{23}$. Le lieutenant-colonel C. G. Yersin de Payerne 


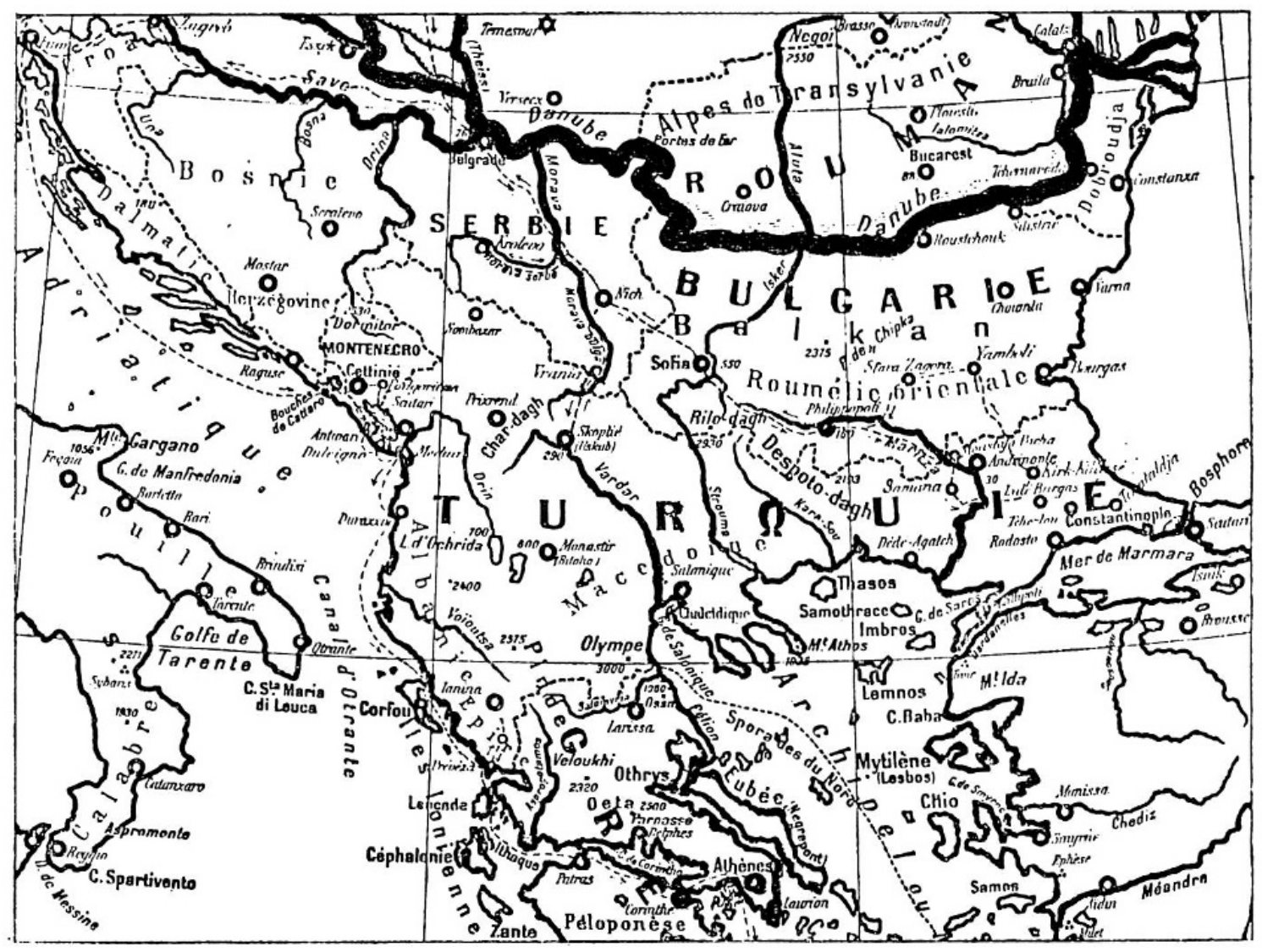

Fig. 2. Les pays des Balkans en 1912 - avant les changements territoriaux provoqués par la première guerre des Balkans (1912). La carte a fait part du rapport du Dr. C. de Marval au Comité International de la Croix-Rouge

fut l'auteur d'une publication remarquée et bien documentée ${ }^{24}$. En 1913, il fut l'animateur d'une équipe de quatre médecins partie aux Balkans, munie d'un équipement militaire très complet. Impressionné par la lutte pour la liberté du peuple serbe, Yersin a consacré à l'histoire de ce peuple 17 pages sur les 38 que comportait son livre. Un autre membre de ce groupe, R. Eugène Bourquin, a aussi publié un rapport d'activité ${ }^{25}$.

Le genevois Albert Jentzer (fils du gynécologue-obstétricien Alcide Jentzer, fondateur de la Maternité de Genève), à cette époque encore jeune chirurgien, se rend en 1913 en Serbie accompagné de trois collègues. A Zaječar, une ville de l'est du pays, Jentzer a travaillé trois mois dans les hôpitaux de cette ville où lui était confié tout le travail chirurgical. A son retour, il a donné à l'Aula de l'Université de Genève une conférence qu'il publiera sous forme d'une brochure illustrée ${ }^{26}$. Deux tiers de ce mémoire riche d'informations chirurgicales sont consacrés à l'histoire et aux traditions culturelles serbes. De même que Yersin et qu'un autre 


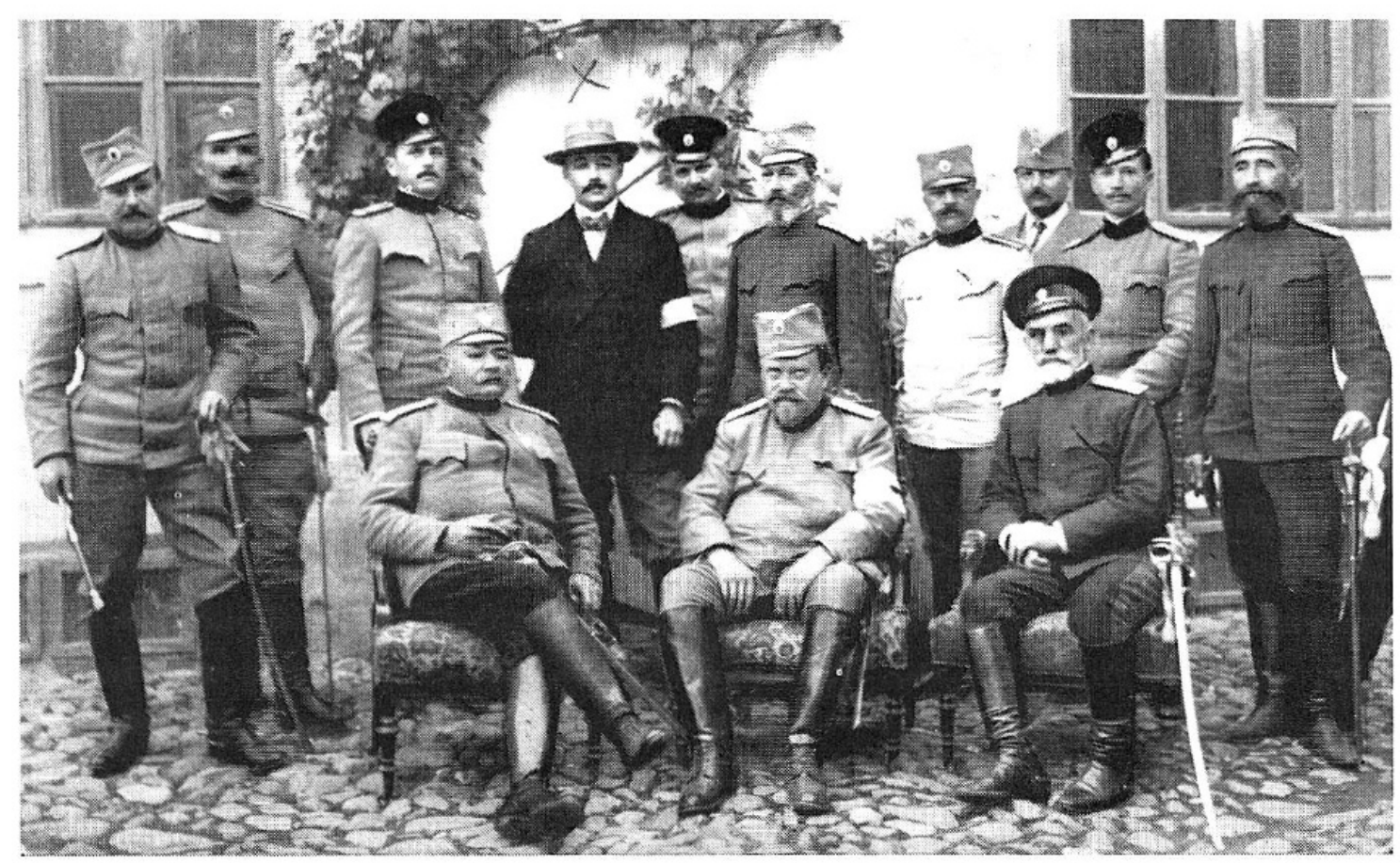

Fig. 3. Le Dr. Albert Jentzer de Genève $(\times)$ avec un groupe d'officiers serbes à Zaječar, en 1912

chirurgien suisse, Adolf Vischer de la clinique du professeur Frédéric de Quervain de Bâle, qui en 1912 était parti en mission en Macédoine avec Ch. Socin ${ }^{27}$ et Eduard Stierlin et qui a publié un livre impressionant à l'intention des lecteurs non-médicaux sur ce voyage ${ }^{28}$, - Jentzer s'était en effet rendu compte que les connaissances de ses concitoyens au sujet de la Serbie étaient pratiquement inexistantes ou erronées. Avec leurs publications, ces trois auteurs ont, après avoir accompli un devoir médical, réussi à accomplir aussi une mission culturelle et politique. Au cours de la Première Guerre Mondiale, Jentzer (qui en 1933 va être élu professeur et devenir un des grands patrons de la chirurgie genevoise) a publié des observations très positives sur une nouvelle méthode d'extension applicable aux fractures de la jambe, inventée par le Croate Florschütz; cette méthode l'avait frappé déjà lors de son précédent séjour en Serbie ${ }^{29}$.

De nombreux membres d'autres expéditions médicales venaient de la Suisse alémanique. Une importante mission était envoyée à Belgrade et a travaillé partiellement après en Albanie. Elle était sous la conduite de Paul Niehans. Parmi les infirmières se trouvaient Alice Wirz, Nadia Jaccard et Ida Huth de l'institution des Dames Genevoises de la Croix-Rouge. Une autre infirmière, Louise Probst de Bâle, a décrit cette expédition avec un sens extraordinaire d'objectivité critique dans une brochure ${ }^{30}$. Comme il avait été le cas avec les directeurs des cliniques 
chirurgicales de Lausanne, Genève et Bâle, le Prof. Theodor Kocher de Berne a aussi encouragé la participation de ses assistants dans les services volontaires aux Balkans. Il a montré son vif intérêt pour les nouvelles acquisitions de la chirurgie de guerre. (Comme J.-L. Reverdin de Genève, Kocher a pendant des décennies enseigné la chirurgie militaire aux jeunes médecins suisses.) Kocher prit une part active dans la discussion ${ }^{31}$ après les communications de ses assistants, C.A.Pettavel $^{32}$ et F. Rusca ${ }^{33}$, sur leurs exploits en Bulgarie et en Serbie. Dans la série de ces comptes rendus, il faut citer encore ceux de K. Reber ${ }^{34}$, d'Eduard Stierlin et Adolf Vischer ${ }^{35}$, enfin de Ch. Socin ${ }^{27}$.

Ce n'etaient pas seulement des chirurgiens qui étaient attirés par les services volontaires. Louis Michaud, qui deviendra directeur de la Clinique médicale de Lausanne, à ce moment-là encore assistant à Kiel (Allemagne du Nord), a publié ses observations sur les maladies infectieuses en Serbie dans la Revue médicale de la Suisse romande ${ }^{36}$.

Pendant la Grande Guerre de 1914-1918, de nombreux médecins suisses, individuellement ou en groupe, s'engagent non seulement dans les Balkans, mais aussi dans d'autres pays, surtout en France. Le colonel Hauser, médecin-en-chef de l'armée suisse, entreprend de nombreux voyages d'information en Allemagne et en France. Il les complète par les renseignements que lui fournissent les médecins suisses au retour de leurs missions. Ces expériences vont faciliter l'élaboration d'une doctrine médicale militaire adaptée aux besoins spécifiques du pays ${ }^{4}$.

C'est dans une optique plus large, internationale, qu'il faut apprécier quelques autres expériences et publications. C'est le cas, par exemple, avec le livre du Lausannois M. Jeanneret-Minkine sur le typhus exanthématique, vécu et étudié en Serbie, paru à Paris en $1915^{37}$. Cette œuvre était lue et appréciée dans le monde entier : la preuve en est que plusieurs pages sont entièrement citées dans le livre classique sur le typhus publié en 1920 par les Américains Strong et Zinsser ${ }^{38}$. L'engagement médical volontaire en temps de guerre a parfois permis des recherches et des découvertes scientifiques. Dans le contexte de notre thème, l'exemple était donné par Ludwig Hirszfeld (d'origine polonaise), privat-docent en microbiologie à Zurich. Il s'est rendu en compagnie de sa femme Hanna (assistante du professeur de pédiatrie Emil Feer) à deux reprises en 1914 et 1915-1918 en Serbie et à Salonique. Après avoir participé au combat contre le typhus, ils ont passé par le calvaire de la retraite de l'armée serbe en Albanie. A Salonique, Hirszfeld a réussi à découvrir un nouveau type - C - de la parathyphoïde. De plus, la présence simultanée de troupes des différentes nations lui a fourni les circonstances favorables à la création d'une nouvelle discipline scientifique : l'épidémiologie des groupes sanguins - soit la sérologie anthropologique ${ }^{39,40}$. 
Mentionnons un cas tout-à-fait spécial : l'engagement professionnel et humain dans les souffrances de la population serbe du criminologue lausannois Rudolf Archibald Reiss; après la guerre, il a décidé de s'installer à Belgrade. Du fait que ses activités entre 1916 et 1918 appartiennent au domaine de la médecine légale ${ }^{41}$, une description, même brève, de son rôle dépasse les limites de notre étude.

Retournons à Genève en 1914. Un médecin enthousiaste et courageux, Victor Kühne, qui s'était déjà rendu en Serbie en 1913 et qui a publié ses expériences sur un nouveau traitement du choléra ${ }^{42}$, est en train de former une nouvelle équipe médicale pour la Serbie, dont font partie sa femme et sept autres médecins. Deux entre eux, la doctoresse Eva Mitnitskaia et le docteur Stempelberg, vont payer de leur vie leur dévouement, puisqu'ils seront victimes de l'épidémie du typhus. Les autres membres du groupe Kühne étaient le Brésilien Cenobelino de Barros Serra, Catherine Levkoff-Gonzenberg, Chava Tamarina (Polonaise), F.Paccoud de Lausanne et Oscar Hug de Lucerne, ancien assistant du professeur César Roux de Lausanne. Mentionnons, parmi les nombreux autres volontaires, encore le docteur Hans Hermann Peyer de Schaffhouse, parce qu'il se trouvait déjà pour la seconde fois en mission dure et presque solitaire au Monténégro où il faillit succomber au typhus ${ }^{43}$ a,b. Cette maladie est aussi le leit-motiv du livre-mémoires de H. Vogel ${ }^{44}$.

Notre dernier chapitre concernant la deuxième Guerre Mondiale, qui pour la Yougoslavie a commencé en printemps 1941, sera relativement court, car ce ne fut pas avant la phase finale de la guerre qu'une équipe de médecins suisses est partie sur le territoire libéré par les partisans de Tito, lesquels ont déjà commencé la poursuite des divisions allemandes en retraite. En octobre 1944, après l'intervention des conseillers fédéraux Celio et Nobs, les autorités ont donné le feu vert pour le départ de cette première mission à laquelle appartenaient - sous la conduite chirurgicale du Zurichois Guido Piderman - le Genevois Marc Oltramare, le Tessinois Elio Canevascini, le psychiatre zurichois Paul Parin, le docteur Auguste Matthey de Zurich, sa sœur Liselotte Matthey (aujourd'hui Madame Parin) et un réfugié allemand, le Dr Hannes Merbeck. L'armée suisse a fourni à cette mission l'équipement complet pour un hôpital de campagne. Pour atteindre son lieu de destination au Monténégro, la mission a pris une route plus longue et a mis plus de temps que la mission de Ferrière et de ses collègues en 1875, parce qu'elle a dû passer par les régions libérées de la France et de l'Italie du Sud avant de traverser l'Adriatique. De nombreux témoignages personnels rendus sur les lieux pendant la guerre, puis les publications de trois des membres de cette mission constituent les preuves des services précieux qu'elle a rendus dans le cadre des équipes chirurgicales mobiles et des hôpitaux improvisés ${ }^{45}, 46,47,48$. 


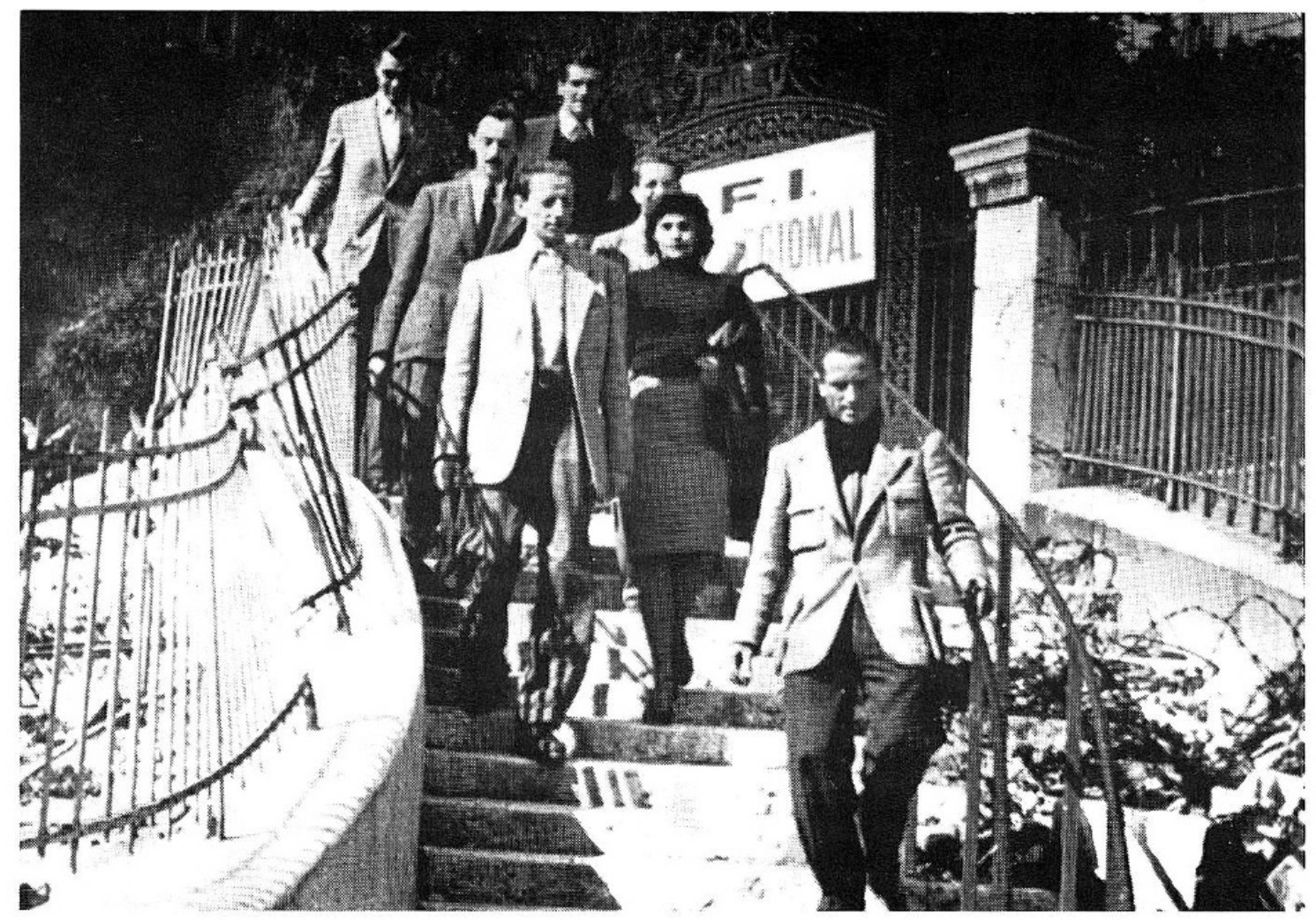

Fig. 4. Les membres de la mission médicale suisse («groupe Piderman») avant leur départ de Suisse pour la Yougoslavie, octobre 1944

Avant la fin de la guerre, le groupe de Piderman a été renforcé par la Zurichoise Elisabeth Vœgeli-Diem. Une mission spéciale pour la Jutte contre le typhus a été conduite par le célèbre expert professeur Hermann Mooser de Zurich ; une mission pédiatrique, par le Dr Rudolf Büeler; une mission orthopédique, par le professeur H. U.Buff de Zurich; une, enfin, de médecine vétérinaire, par le Dr Helmuth Kind de Zurich ${ }^{49}$.

En guise de conclusion, on peut affirmer que la contribution des médecins et des infirmières de Genève, des cantons romands et de la Suisse entière, aux services de santé militaires et civils yougoslaves et des autres peuples balkaniques témoignent d'un esprit de dévouement, et de la dignité apportée dans l'accomplissement d'une tâche professionnelle et humaine. De retour à la vie «normale», ils ont transmis leurs expériences sur un plan local et national, souvent même mondial, soit dans le cadre du travail quotidien, soit sous forme des conférences et publications. Quelques uns ont aussi essayé de répandre des connaissances générales, géographiques, historiques et ethnologiques au sujet des peuples des Balkans. De tous ces hommes et femmes, on peut dire - en se servant d'une phrase d'Albert Jentzer ${ }^{50}$-, qu'ils étaient des «soldats de la paix». 


\section{Bibliographie}

${ }^{1}$ Löwenthal, Z., Beiträge zur Geschichte der schweizerisch-jugoslawischen medizinischen Beziehungen. Verhandlungen des XIX. Internationalen Kongresses für Geschichte der Medizin (Basel 1964), Basel/New York 1966, p.452-460.

${ }^{2}$ Levental, Z., Ratnici i ranjenici. Sećanja stranih lekara iz naših ratova. Med. Glas. 17 (1963), Separat p.1-12.

${ }^{3}$ Levental, Z., Neki aspekti istraživanja iskustva stranih lekara u jugoslovenskim zemljama. Zbornik I. Kongr. ist. med. Sarajevo, 1970, p. 87-101.

${ }^{4}$ Hauser (Colonel), Exposé sur les enseignements tirés de la guerre mondiale et appliqués au service de santé de notre armée. Revue médicale de la Suisse romande 35 (1915), p. 913-943.

${ }^{5}$ Ador, G., et Moynier, G., Les destinées de la Convention de Genève pendant la Guerre de Serbie, Genève 1876.

${ }^{6}$ Wepf, R., Das Abenteuer Helfen. Als Rotkreuzdelegierter und Chirurg in 10 Kriegsländern. Bern 1973.

${ }^{7}$ Rapport sur l'intervention de la Croix-Rouge Suisse pendant la guerre des Balkans, Berne 1913.

${ }^{8}$ Humbert, A., Ferrière, F., et Goetz, Ch., Mission en Monténégro. Bull. Int. des Soc. de Sec. aux Milit. Blessés 7 (Genève 1876), p. 55-63.

${ }^{9}$ Ferrière, F., Le Monténégro. Notes géographiques et souvenirs de voyages. Le Globe (Genève), $1^{\text {re }}$ partie : 20 (1881), p. 73-99; II ${ }^{\mathrm{e}}$ partie: 21 (1882), p.93-124.

${ }^{10}$ Ferrière, Adolphe, Le Dr Frédéric Ferrière, Genève 1948.

11 A.R.; P.D.G.; G.W., Dr Frédéric Ferrière, 1848-1924. Extrait de la Revue Int. et Bull. Int. des Soc. de la Croix-Rouge 55 (1924), p.1-64.

12 C.P., Le Dr Frédéric Ferrière (1848-1924). Nécrologie. Revue médicale de la Suisse romande 44 (1924), p. 537-542.

${ }^{13}$ Ferrière, F., L'assistance aux prisonniers dans la guerre des Balkans. Bull. Int. des Soc. de la Croix-Rouge 44 (1913), p. 200-212.

${ }^{14}$ Ferrière, F., Quelques constatations sur les conditions sanitaires dans la guerre des Balkans. Bull. Int. des Soc. de la Croix-Rouge 44 (1913), p.212-216.

${ }^{15}$ Ferrière, F., Guerre des Balkans. - Questions sanitaires. Bull. Int. des Soc. de la CroixRouge 44 (1913), p.284-293.

${ }^{16}$ Rolland, Romain, Inter arma caritas, in: Au-dessus de la mêlée, Paris/Neuchâtel 1915.

${ }^{17}$ Zweig, Stefan, Le Cœur de l'Europe. Une visite à la Croix-Rouge Internationale de Genève. Genève/Paris 1918.

${ }^{18}$ La guerre des Balkans. Bull. Int. des Soc. de la Croix-Rouge 44 (1913), p.24-62.

${ }^{19}$ La guerre des Balkans. Bull. Int. des Soc. de la Croix-Rouge 44 (1913), p.136-145.

${ }^{20}$ Sturzenegger, Catharina, Serbisches Rotes Kreuz und internationale Liebestätigkeit während der Balkankriege 1912/1913, Zürich 1914.

${ }^{21}$ Reverdin, A., L'activité de l'ambulance Vaud-Genève pendant la guerre gréco-turque. Bull. Int. des Soc. de la Croix-Rouge 44 (1914), p.657-676.

${ }^{22}$ Matthey, Alfred-C., Dans les Balkans. Serbie et Constantinople. Notes de chirurgie de guerre. Campagne de 1912-13. Revue médicale de la Suisse romande 33 (1913), p. 297-308 et p. 388-397.

${ }^{23}$ Marval, Carle de, Rapport sur sa mission dans les Balkans. Bull. Int. des Soc. de la CroixRouge 44 (1913), p.17-24. 
24 Yersin, C. G., Compte rendu chirurgical de la Campagne Serbo-Bulgare 1913 (Précédé d'un court aperçu historique), Genève 1917.

${ }^{25}$ Bourquin, R.E., Deux mois en Serbie pendant la seconde guerre balkanique. Revue médicale de la Suisse romande 34 (1914), p.100-110.

${ }^{26}$ Jentzer, A., Petite étude sur la Serbie. Impressions et souvenirs recueillis pendant la guerre turco-balkanique. Genève 1914.

${ }^{27}$ Socin, Ch., Beobachtungen über den serbischen Heeressanitätsdienst. Corr.-Blatt für Schweizer Ärzte 43 (1913), p.1-7 (Militärärztliche Beilage).

${ }^{28}$ Vischer, A., An der serbischen Front. Erlebnisse eines Arztes auf dem serbisch-türkischen Kriegsschauplatz 1912. Basel 1913.

${ }^{29}$ Jentzer, A., De la méthode serbo-croate pour réduire les fractures du fémur et de la jambe. Revue médicale de la Suisse romande 35 (1915), p.450-454.

30 Probst, Schwester Louise, Erinnerungen an die Schweizerische Rotkreuzexpedition nach Serbien, Basel 1913.

${ }^{31}$ Kocher, Th., Diskussionsteilnahme. Corr.-Blatt für Schweizer Ärzte 43 (1913), p. 914-915.

32 Pettavel, C.A., Chirurgische Erfahrungen aus dem Balkankriege (Bulgarien). Corr.-Blatt für Schweizer Ärzte 43 (1913), p.929-941.

${ }^{33}$ Rusca, F., Erfahrungen im Balkankrieg. I. Die Ursachen der Infektionen. II. Kasuistische Mitteilungen. Corr.-Blatt für Schweizer Ärzte 43 (1913), p.1689-1698, 1740-1748.

${ }^{34}$ Reber, K., Kriegschirurgische Erfahrungen im Balkankriege. Corr.-Blatt für Schweizer Ärzte 44 (1914), p.1494-1508.

${ }^{35}$ Stierlin, E., und Vischer, A., Erfahrungen mit dem Mastisolverband im serbisch-türkischen Krieg. Corr.-Blatt für Schweizer Ärzte 43 (1913), p. 588-591.

${ }^{36}$ Michaud, L., Observations sur les maladies infectieuses pendant la guerre des Balkans. Revue médicale de la Suisse romande 34 (1914), p.552-556.

37 Jeanneret-Minkine, M., Le typhus exanthématique, Paris 1915.

${ }^{38}$ Strong, R.P., Zinsser, H., et al., Typhus fever with particular reference to the Serbian epidemic, Cambridge (USA) 1920.

${ }^{39}$ Hirszfeld, L., Aus meinen Erfahrungen als Hygieniker in Serbien. Corr.-Blatt für Schweizer Ärzte 46 (1916), p.513-531.

${ }^{40}$ Hirszfeld, L., Historià jednego žycia, Warszawa 1946.

${ }^{41}$ Reiss, R.A., Lettres du front macédono-serbe 1916-1918, Genève 1921.

${ }^{42}$ Kuhne, V., Que faire en cas d'épidémie de choléra? (Une médication causale du syndrome diarrhéique.) Revue médicale de la Suisse romande 38 (1918), p.555-569.

43 a Nagel, E., Die Liebestätigkeit der Schweiz im Weltkriege, 2 Bände, Basel 1916.

43 b Nagel, E., Les œuvres suisses de charité pendant la guerre 1914-1918, 2 vol., Neuchâtel 1916, vol. II, p.90-93.

${ }^{44}$ Vogel, H., Valjevo. Erinnerungen eines Schweizer Arztes an den serbisch-türkischen Krieg, Rorschach 1941.

45 Piderman, G., und Parin, P., Zur Behandlung schwerer Extremitätsverletzungen. Therapeutische Umschau 3 (1946), Separat p.1-8.

${ }^{46}$ Parin, P., Die Kriegsneurosen der Jugoslawen. Schw. Arch.f. Neur. u. Psych. 41 (1948), Separat p.1-24.

${ }^{47}$ Merbeck, H., Erinnerungen an die chirurgische Tätigkeit bei den jugoslawischen Partisanen 1944-1945. Unveröffentlichtes Manuskript. 
48 Merbeck, H., Kao ratni hirurg u NOV Jugoslavije. Vojnosanit. preg. 4 (1948), p.171-179.

${ }^{49}$ Piderman, G., Persönliche schriftliche Mitteilung an Z. Levental, 1962.

${ }^{50}$ Jentzer, A., Conférence inaugurale en fonction de professeur en chirurgie, Genève 1933.

En 1962, une de mes étudiantes de Belgrade, Gordana Djordjević, a publié - avec mon assistance - sur les missions suisses dans les guerres de peuples yougoslaves un article sous le titre «Švajcarske sanitetske misije u našim ratovima» dans la Revue éstudiantine Medicinski podmladak 14. (1962/63), p. 81-88.

\section{Summary}

A chronological survey on the role and the characteristics of Swiss medical missions in the liberation wars of the Yugoslav peoples is given, and various reasons for this involvement are explained. The first Swiss medical mission has been sent by the International Committee of the Red Cross to Montenegro in 1875. One of the three members, Dr.F.Ferrière, has afterwards played an important role in the international Red Cross activities, especially during World War I, when he created the international office for protection of suffering civilians.

During the two Balkan wars 1912/13 more than 80 Swiss doctors and nurses, mainly in Red Cross expeditions, worked for the army medical services of the Balkan countries. The two biggest missions have been A. Reverdin's - from Geneva - to Greece and P. Niehans's from Berne - to Serbia. Also during the War 1914-1918 a great number of Swiss voluntaries rendered precious services to the Serbian and Montenegrinian armies. Some of them lost their lifes as victims of typhus fever. In World War II the first Swiss mission has joined the Yugoslav partisans not before October 1944.

The experiences of all these missions have been an important contribution to the efforts of the Balkan military and civilian health services, but in the same time also for the up to date orientation of Swiss military medicine and surgery in general. On an international level, some of the Swiss publications contributed to a better knowledge especially of typhus fever, as well as to more information and understanding of the Yugoslav peoples' history, culture and ethnology.

The bibliographical data contain the most important articles on the subject in Swiss and other periodicals, as well as a number of books $(6,10,17,20,24,26,28,30,37,38,40,41$ and 44).

Prof. Dr. Zdenko Levental

Institut für Ausbildungs- und Examensforschung der Medizinischen Fakultät der Universität Bern Inselspital $14 \mathrm{c}$

3010 Bern 